\title{
Evaluation of Sixteen Cucumber (Cucumis sativus L.) Genotypes in Derived Savannah Environment Using Path Coefficient Analysis
}

\author{
Chikezie O. ENE*, Peter E. OGBONNA, Christian U. AGBO, Uche P. CHUKWUDI \\ University of Nigeria, Nsukka, Faculty of Agriculture, Department of Crop Science, Enugu State, Nigeria; enechike17@gmail.com ("correspondingauthor), \\ ogbonna1968@gmail.com;christian.agbo@unn.edu.ng; uchechukwu.chukwudi@unn.edu.ng
}

\begin{abstract}
Sixteen exotic and indigenous cucumber genotypes (Cucumis sativus L.) were evaluated under early and late seasons planting. This was to characterize the cucumber genotypes based on their performance in the derived savannah of Southeast Nigeria agro-ecological zone and estimate character association and contribution towards total fruit yield per hectare. The vegetative traits, such as vine length, number of branches, number of leaves and leaf area were measured at 8 weeks after planting. The phonological traits measured were: days to flower initiation and days to $50 \%$ flowering. The reproductive traits like number of staminate flowers per plant, number of pistillate flowers per plant, fruit length, fruit girth, fruit weight per plant, number of fruits per plant, average fruit weight and total fruit yield per hectare were also recorded. Obtained data was subjected to analysis of variance in randomized complete block design and path analysis. The results showed a highly significant difference $(\mathrm{p}<0.01)$ among the genotypes in all the traits studied in both seasons. 'Beit Alpha' genotype gave the highest fruit yield/ha in early season planting, while in the late season, the highest yield producer was 'Ashely'. In both seasons, vine length, number of branches and leaves, leaf area, number of pistillate and staminate flowers/plant, number of fruit and fruit weight/plant showed positive and significant correlation with total fruit yield/ha. In both seasons, the highest positive direct effect on yield was recorded in fruit weight/plant. Significantly $(\mathrm{p}<0.05)$, the highest total fruit yield/ha was recorded in the early rainy season planting in this agro ecological zone. Based on high fruit yield, 'Beit Alpha' genotype for early season planting and 'Ashley' genotype for late season are recommended for cultivation in the Derived Savannah, Southeast Nigeria agro ecological zone.
\end{abstract}

Keywords: characterization, correlation, cucumber, fruit yield, seasonal effects, savannah, agro-ecological zone

\section{Introduction}

Cucumber (Cucumis sativus L.) belongs to the "gourd" family Cucurbitaceae. India had been proposed as the center of origin (Whitaker and Davis, 1996; Renner et al., 2007; Anonymous, 2012). It is an important vegetable crop (Lower and Edwards, 1986; Thoa, 1998; Eifediyi and Remison, 2010) and had being in cultivation since 3,000 years ago (Wehner and Guner, 2004). In Asia, it is the most cultivated vegetable after tomato, cabbage and onion (Tatlioglu, 1993; Wehner, 2007), while in Western Europe, it is second only to tomato (Phu, 1997). In tropical Africa, the crop has not been ranked probably because of insufficient yield and limited use (Eifediyi and Remison, 2009).

The fruit has about 95\% water content (Anonymous, 2012) which makes it diuretic, possessing a deep cleansing action due to the presence of some natural chemical constituents such as glycolic, lactic, and salicylic acids (Eneobong, 2001; Uzodike and Onuoha, 2009). The juice is a valuable medicinal food in the treatment of hyperacidity in gastric and duodenum ulcer, a good laxative for constipation (Ernestina, 2001). Most skin infections had been successfully treated with a cucumber extract, alpha hydroxyl acid (Swanbeck, 1968; Uzodike and Onuoha, 2009).
Cucumber is grown widely in different parts of the world. It is an all year round out door vegetable in the tropics (Mingbao, 1991; Eifediyi and Remison, 2010). Jizhe (1993) and Eifediyi and Remison (2009) opined that cucumber is a typical vegetable of warm temperate and cool tropical areas that can be cultivated at any time of the year. Many crop species have done well in yield in a particular season or location but have failed to perform likewise in some other environments. This suggests that both temporal and spatial environment affect crop yield. This may be due to some climatic or edaphic factors or both. A good understanding of this fact will help breeders to characterize these genotypes based on their performance in a given environment.

Correlation among traits and with yield is important in indirect selection of genotypes for yield improvement (Machikowa and Laosuwan, 2011). Significant and positive correlation between two characters suggests that these characters can be improved simultaneously in a selection programme (Hayes et al., 1955; Nwofia et al., 2015) and selection for one will translate to selection and improvement of the other (Fayeun et al., 2012). However, selection decision based on correlation coefficient alone may give a misleading impression as it only measures the degree of mutual association between two variables without regard to 
86

causation. This is because there is the risk of omitting some useful traits whose contributions through other traits might not be easily appreciated.

The success of any breeding programme depends greatly on the genetic diversity available in a population (Afangideh et al., 2005; Subramanian and Subbaraman, 2010). The variation in the performance of cucumber varieties has been widely studied by many scholars (Manyvong, 1997; Ajisefinanni, 2004) but systematic work to characterize available genotypes based on their variability in Derived savannah agro ecological zone of Southeast Nigeria is scarce. It is against this background that this study was carried out to evaluate the performance of exotic and indigenous cucumber genotypes available in the Southeastern Nigeria agroecological zone and estimate character association and contribution towards total fruit yield/ha.

\section{Materials and Methods}

The experiment was carried out in the Department of Crop Science Research farm, Faculty of Agriculture, University of Nigeria, Nsukka. Nsukka is located on latitude $6{ }^{\circ} 51^{1} \mathrm{E}$, and longitude $7^{\circ} 29^{1} \mathrm{~N}$ of $475 \mathrm{~m}$ above sea level, characterized by lowland humid condition with bimodal annual rainfall distribution that ranges from $1155 \mathrm{~mm}$ to $1955 \mathrm{~mm}$, a mean annual temperature of $29^{\circ} \mathrm{C}$ to $31^{\circ} \mathrm{C}$ and relative humidity that ranges from $69 \%$ to $79 \%$ (Uguru et al., 2011).

Sixteen cucumber genotypes ('Beit Alpha', 'Delilha', 'Zeina', 'Palmetto', 'Straight 8', 'Table green 72', 'Poinsett', 'Centriolo', 'Regal', 'Sumter', 'Ashely', 'Royal F1', 'MarketMore 76', 'W12757', 'Calypso', and 'Marketer' (Table 1) obtained from National Agricultural Extension, Research and Liaison Services (NAERLS), Ahmadu Bello University, Samaru Zaria, were used for the trial.

They were evaluated in early (May to July) and late (September to November) planting seasons of 2014 in a randomized complete block design with 3 replicates. Monthly rainfall distribution, relative humidity and temperature recorded during this period (Table 2).

The field was prepared and demarcated into 3 blocks. Poultry droppings at the rate of 10 metric tons per hectare were worked into the soil within each block. Each block measuring 3 $\mathrm{m} \times 40 \mathrm{~m}$ contained sixteen plots $(3 \mathrm{~m} \times 2 \mathrm{~m}$ each) for each of the genotypes. Seeds were planted at the spacing of $0.5 \mathrm{~m}$ intra and $0.5 \mathrm{~m}$ inter rows to give a plant population of 40,000 stands per hectare. Two seeds were sown at the depth of $3-4 \mathrm{~cm}$ and were thinned down to a seedling 3 weeks after emergence (WAE). NPK fertilizer in the ratio of 20:10:10 was applied at 2 and 5 WAE at the rate of $300 \mathrm{~kg} / \mathrm{ha}$ after manual weeding in each case. Insecticide (Cypermethrin 110\% EC sprayed at the rate of 125 $\mathrm{ml}$ in 15 litres of water) and fungicide (Maneb Mancozeb and zoxamide at the rate of $75 \mathrm{ml}$ in 15 litres of water) were applied twice ( 2 and $4 \mathrm{WAE}$ ) to curtail insect attack and disease incidence on young plants.

The vegetative traits such as vine length, number of branches, number of leaves and leaf area were measured at 8 weeks after planting. The phonological and reproductive traits like days to flower initiation, days to $50 \%$ flowering, number of staminate flowers per plant, number of pistillate flowers per plant, fruit length, fruit girth, fruit weight per plant, number of fruits per plant, average fruit weight and total fruit yield per hectare were measured.
Table 1. Origins and source of cucumber collection for the carried out trial

\begin{tabular}{llll}
\hline S/N & \multicolumn{1}{c}{ Genotypes } & \multicolumn{1}{c}{ Origin } & \multicolumn{1}{c}{ Remark } \\
\hline 1 & 'Zeina' & NIHORT & Indigenous \\
2 & 'Delilha' & NIHORT & Indigenous \\
3 & 'Beit Alpha' & NIHORT & Indigenous \\
4 & 'Calypso' & CBS, NCSU, U. S.A. & Exotic \\
5 & 'Regal' & CBS, NCSU, U. S.A. & Exotic \\
6 & 'Royal F1' & CBS, NCSU, U. S.A. & Exotic \\
7 & 'Centriolo' & CBS, NCSU, U. S.A. & Exotic \\
8 & 'Ashely' & CBS, NCSU, U. S.A. & Exotic \\
9 & 'Straight 8' & CBS, NCSU, U. S.A. & Exotic \\
10 & 'Sumter' & CBS, NCSU, U. S.A. & Exotic \\
11 & 'W12757' & CBS, NCSU, U. S.A. & Exotic \\
12 & 'Tablegreen 72' & CBS, NCSU, U. S.A. & Exotic \\
13 & 'MarketMore 76' & CBS, NCSU, U. S.A. & Exotic \\
14 & 'Poinsett' & CBS, NCSU, U. S.A. & Exotic \\
15 & 'Marketer' & CBS, NCSU, U. S.A. & Exotic \\
16 & 'Palmetto' & CBS, NCSU, U. S.A. & Exotic \\
\hline NIHORT-Nigerian Horticultural Research Center Ibadan, Nigeria. \\
CBS-Cucumber Breeding Station & & \\
\multicolumn{2}{l}{ NCSU, USA-North Carolina State University, United States of America. }
\end{tabular}

Table 2. Mean monthly rainfall $(\mathrm{mm})$, temperature $\left({ }^{\circ} \mathrm{C}\right)$ and relative humidity (\%) during the early and late season planting of 2014

\begin{tabular}{|c|c|c|c|c|c|}
\hline \multirow[b]{2}{*}{ Month } & \multirow[b]{2}{*}{$\begin{array}{c}\text { Rainfall } \\
(\mathrm{cm})\end{array}$} & \multicolumn{2}{|c|}{ Temperature $\left({ }^{\circ} \mathrm{C}\right)$} & \multicolumn{2}{|c|}{ Relative Humidity (\%) } \\
\hline & & Min & $\operatorname{Max}$ & $10 \mathrm{am}$ & $4 \mathrm{pm}$ \\
\hline April & 105.16 & 22.30 & 31.30 & 69.93 & 70.53 \\
\hline May & 241.14 & 21.06 & 28.29 & 72.26 & 72.26 \\
\hline June & 271.79 & 20.87 & 29.13 & 72.00 & 72.00 \\
\hline July & 195.81 & 20.90 & 27.74 & 72.19 & 72.19 \\
\hline August & 92.36 & 20.71 & 27.29 & 73.00 & 73.00 \\
\hline September & 401.99 & 20.33 & 27.90 & 73.00 & 73.00 \\
\hline October & 211.08 & 20.84 & 28.90 & 73.00 & 72.77 \\
\hline November & 77.22 & 21.00 & 30.07 & 73.80 & 71.97 \\
\hline December & 4.83 & 19.03 & 30.65 & 70.58 & 70.06 \\
\hline
\end{tabular}

Source: Meteorological Station, Department of Crop Science, University of Nigeria, Nsukka

\section{Statistical analysis}

The data collected were subjected to analysis of variance to show the level of variability among the cucumber genotypes. The significant means were separated using the F-LSD procedure (Obi, 2002). Pearson correlation coefficient analysis was done using the computer statistical software package, SPSS version 16. The sets of correlation coefficients were subjected to path coefficient analysis and the direct and indirect effects were estimated according to the method of Dewey and Lu (1959) to show the relationships between traits and contribution towards total fruit yield/ha in cucumber genotypes.

\section{Results}

The result of the meteorological data (Table 2) showed that the highest rainfall $(\mathrm{cm})$ was recorded in September in late season planting. However, early season showed even distribution of rainfall unlike in late season. Temperature $\left({ }^{\circ} \mathrm{C}\right)$ and relative humidity (\%) showed relatively uniform distribution in both seasons. The performance of cucumber genotypes with respect to some agronomic traits evaluated in 2014 early season planting is presented in Table 3. The result showed a significant $(\mathrm{p}<0.05)$ 
Table 3. Performance of cucumber genotypes with respect to some agronomic traits evaluated in 2014 early season planting

\begin{tabular}{|c|c|c|c|c|c|c|c|c|c|c|c|c|c|c|c|c|}
\hline Accessions & $\begin{array}{c}\text { VL8 } \\
\text { WAP }\end{array}$ & $\begin{array}{l}\text { NoB8 } \\
\text { WAP }\end{array}$ & $\begin{array}{l}\text { NoL8 } \\
\text { WAP }\end{array}$ & $\begin{array}{c}\text { LA8 } \\
\text { WAP }\end{array}$ & DMFI & DFFI & $\begin{array}{l}\mathrm{D} 50 \% \\
\mathrm{MF}\end{array}$ & $\begin{array}{c}\text { D50\% } \\
\text { FF }\end{array}$ & $\begin{array}{c}\text { NoSF } \\
\text { PP }\end{array}$ & $\begin{array}{l}\text { NoPF } \\
\text { PP }\end{array}$ & $\begin{array}{l}\mathrm{NoF} \\
\mathrm{PP}\end{array}$ & FL & FG & $\begin{array}{l}\text { FW } \\
\text { PP }\end{array}$ & AFW & TFY/ha \\
\hline 'Zeina' & 27.89 & 0.00 & 15.11 & 70.24 & 26.67 & 36.33 & 34.33 & 43.33 & 9.48 & 2.58 & 4.73 & 15.12 & 15.81 & 0.64 & 0.22 & 6.42 \\
\hline 'Palmetto' & 30.09 & 0.00 & 10.47 & 47.95 & 27.33 & 38.00 & 35.67 & 46.00 & 8.80 & 2.10 & 3.31 & 12.97 & 13.15 & 0.56 & 0.17 & 5.63 \\
\hline 'Centriolo' & 215.28 & 4.00 & 39.28 & 111.79 & 28.00 & 37.67 & 35.67 & 45.67 & 14.07 & 4.21 & 9.16 & 19.60 & 17.35 & 0.97 & 0.11 & 9.70 \\
\hline 'W12757' & 22.98 & 0.00 & 9.21 & 36.72 & 30.33 & 40.00 & 39.67 & 47.67 & 7.77 & 2.06 & 3.58 & 10.73 & 10.71 & 0.42 & 0.13 & 4.27 \\
\hline 'Beit Alpha' & 283.91 & 4.36 & 34.09 & 79.45 & 23.00 & 31.67 & 31.67 & 40.33 & 14.99 & 4.56 & 10.49 & 21.33 & 20.26 & 2.00 & 0.19 & 20.24 \\
\hline 'Ashely' & 299.72 & 4.35 & 41.79 & 170.83 & 23.00 & 32.00 & 31.33 & 40.00 & 17.48 & 5.30 & 11.62 & 21.15 & 19.71 & 1.98 & 0.17 & 19.81 \\
\hline 'Marketer' & 104.94 & 2.33 & 22.34 & 57.66 & 27.33 & 37.67 & 35.67 & 47.00 & 7.58 & 1.91 & 2.61 & 12.68 & 13.08 & 0.75 & 0.29 & 7.54 \\
\hline 'Poinsett' & 241.27 & 2.78 & 37.55 & 92.81 & 33.67 & 43.33 & 42.00 & 52.00 & 9.38 & 2.51 & 2.95 & 17.18 & 16.80 & 0.96 & 0.32 & 9.61 \\
\hline 'Straight 8' & 296.27 & 4.21 & 30.47 & 156.03 & 24.67 & 35.00 & 32.33 & 41.00 & 19.46 & 6.87 & 11.97 & 22.17 & 20.34 & 2.01 & 0.17 & 20.00 \\
\hline 'Tablegreen 72 ' & 203.84 & 3.07 & 34.44 & 141.01 & 24.00 & 34.33 & 33.00 & 42.00 & 16.64 & 4.79 & 9.00 & 17.69 & 16.60 & 1.20 & 0.14 & 12.01 \\
\hline 'Regal' & 165.46 & 3.08 & 29.27 & 173.30 & 23.00 & 32.33 & 31.00 & 39.00 & 17.37 & 4.82 & 10.48 & 15.47 & 15.81 & 0.70 & 0.07 & 7.00 \\
\hline 'Delilha' & 80.20 & 2.36 & 14.79 & 29.29 & 34.67 & 44.33 & 43.33 & 51.67 & 8.54 & 2.10 & 2.59 & 10.12 & 9.80 & 0.70 & 0.27 & 7.04 \\
\hline 'Sumter' & 259.1 & 4.08 & 45.15 & 178.64 & 23.67 & 33.67 & 32.67 & 50.00 & 19.67 & 6.55 & 11.92 & 20.80 & 19.30 & 1.68 & 0.14 & 16.86 \\
\hline 'RoyalF1' & 18.41 & 0.00 & 10.39 & 28.73 & 36.00 & 45.67 & 44.00 & 54.33 & 9.30 & 2.16 & 2.77 & 11.45 & 10.52 & 0.18 & 0.22 & 1.89 \\
\hline MarketMore76 & 19.88 & 0.00 & 8.41 & 27.61 & 35.67 & 45.67 & 44.00 & 53.67 & 8.89 & 2.05 & 2.51 & 9.56 & 10.05 & 0.21 & 0.09 & 2.19 \\
\hline 'Calypso' & 29.54 & 0.00 & 14.23 & 32.49 & 35.33 & 45.67 & 45.00 & 54.33 & 10.53 & 2.35 & 2.77 & 11.24 & 11.15 & 0.35 & 0.13 & 3.52 \\
\hline Mean & 143.67 & 2.16 & 24.81 & 89.66 & 28.52 & 38.33 & 36.96 & 46.75 & 12.50 & 3.56 & 6.40 & 15.58 & 15.03 & 0.96 & 0.18 & 9.61 \\
\hline F-LSD 0.05 & 5.73 & 1.21 & 2.91 & 9.21 & 1.58 & 2.19 & 1.75 & 1.26 & 1.26 & 0.67 & 0.97 & 1.34 & 1.22 & 0.26 & 0.14 & 0.09 \\
\hline
\end{tabular}

VL8WAP=Vine length at 8 weeks after planting $(\mathrm{cm}), \mathrm{LA} 8 \mathrm{WAP}=$ Leaf area at 8 weeks after planting $\left(\mathrm{cm}^{2}\right)$, NoB8WAP=Number of branches at 8 weeks after planting, NoL8WAP=Number of leaves at 8 weeks after planting, DMFI=Days to male flower initiation, DFFI=Days to female flower initiation, D50\%MF=Days to $50 \%$ male flowering, $\mathrm{D} 50 \% \mathrm{FF}=$ Days to $50 \%$ female flowering, NoPFPP=Number of pistillate flower per plant, NoSFPP=Number of staminate flower per plant, FG=Fruit Girth (cm), FL=Fruit length (cm), FWPP=Fruit weight per plant $(\mathrm{kg}), \mathrm{AFW}=$ Average fruit weight $(\mathrm{kg}), \mathrm{NoFPP}=\mathrm{Number}$ of fruit per plant, TFY/Ha=Total fruit yield per hectare (tons/ha)

Table 4. Performance of cucumber genotypes with respect to some vegetative and yield traits evaluated in 2014 late planting season

\begin{tabular}{|c|c|c|c|c|c|c|c|c|c|c|c|c|c|c|c|c|}
\hline Genotypes & $\begin{array}{c}\text { VL8 } \\
\text { WAP }\end{array}$ & $\begin{array}{l}\text { NoB8 } \\
\text { WAP }\end{array}$ & $\begin{array}{l}\text { NoL8 } \\
\text { WAP }\end{array}$ & $\begin{array}{c}\text { LA8 } \\
\text { WAP }\end{array}$ & DMFI & DFFI & $\begin{array}{c}\text { D50\% } \\
\text { MF }\end{array}$ & $\begin{array}{c}\text { D50\% } \\
\text { FF }\end{array}$ & $\begin{array}{c}\text { NoSF } \\
\text { PP }\end{array}$ & $\begin{array}{l}\text { NoPF } \\
\text { PP }\end{array}$ & NoFPP & FL & FG & $\begin{array}{l}\text { FW } \\
\text { PP }\end{array}$ & MFW & TFY/ha \\
\hline 'Zeina' & 17.70 & 0.00 & 10.38 & 43.06 & 28.67 & 38.67 & 36.67 & 46.33 & 6.91 & 1.03 & 4.34 & 14.91 & 14.83 & 0.33 & 0.08 & 3.31 \\
\hline 'Palmetto' & 16.78 & 0.00 & 8.06 & 30.94 & 29.33 & 40.33 & 37.67 & 48.67 & 6.08 & 0.85 & 2.96 & 12.72 & 13.44 & 0.26 & 0.09 & 2.64 \\
\hline 'Centriolo' & 197.34 & 4.00 & 36.21 & 133.06 & 30.00 & 40.00 & 38.33 & 48.33 & 13.20 & 3.27 & 8.43 & 17.85 & 16.26 & 1.45 & 0.17 & 14.55 \\
\hline 'W12757' & 13.90 & 0.00 & 7.00 & 29.70 & 32.67 & 42.67 & 41.33 & 50.33 & 5.39 & 0.74 & 2.47 & 11.29 & 11.60 & 0.24 & 0.07 & 2.43 \\
\hline 'Beit Alpha' & 197.47 & 3.67 & 34.40 & 137.03 & 24.33 & 33.33 & 33.00 & 41.00 & 14.31 & 3.56 & 8.41 & 18.49 & 17.82 & 1.56 & 0.18 & 15.69 \\
\hline 'Ashely' & 280.03 & 4.35 & 39.92 & 170.97 & 24.33 & 33.33 & 33.00 & 41.67 & 16.68 & 4.16 & 9.56 & 21.29 & 19.40 & 2.01 & 0.21 & 20.10 \\
\hline 'Marketer' & 93.93 & 2.33 & 21.53 & 57.70 & 29.33 & 39.67 & 38.00 & 47.67 & 7.54 & 1.28 & 4.75 & 12.91 & 12.17 & 0.40 & 0.09 & 4.08 \\
\hline 'Poinsett' & 223.60 & 2.78 & 35.96 & 68.52 & 36.00 & 45.67 & 44.33 & 53.00 & 8.55 & 1.75 & 5.71 & 13.22 & 13.03 & 0.65 & 0.11 & 6.51 \\
\hline 'Straight 8 ' & 279.29 & 3.51 & 27.28 & 100.02 & 26.67 & 37.00 & 34.67 & 44.33 & 10.74 & 2.37 & 7.11 & 15.48 & 15.24 & 0.99 & 0.13 & 9.96 \\
\hline 'Tablegreen 72' & 193.70 & 3.07 & 27.44 & 92.39 & 26.00 & 36.33 & 34.33 & 44.67 & 10.28 & 1.97 & 6.99 & 15.20 & 15.21 & 0.80 & 0.11 & 8.07 \\
\hline 'Regal' & 154.75 & 3.08 & 26.50 & 121.44 & 25.00 & 34.33 & 33.67 & 42.33 & 12.28 & 2.81 & 7.77 & 16.60 & 16.13 & 1.25 & 0.15 & 12.52 \\
\hline 'Delilha' & 70.93 & 2.36 & 11.51 & 35.48 & 37.00 & 47.00 & 46.00 & 54.33 & 5.82 & 0.82 & 3.49 & 10.34 & 10.14 & 0.26 & 0.08 & 2.63 \\
\hline 'Sumter' & 243.67 & 4.08 & 40.13 & 145.98 & 25.67 & 35.67 & 34.33 & 47.33 & 14.23 & 3.28 & 8.92 & 18.17 & 16.58 & 1.58 & 0.17 & 15.85 \\
\hline 'Royal F1' & 11.27 & 0.00 & 7.19 & 21.18 & 38.00 & 47.67 & 47.00 & 55.67 & 5.41 & 0.76 & 2.23 & 9.83 & 9.28 & 0.20 & 0.09 & 2.08 \\
\hline MarketMore 76 & 10.51 & 0.00 & 6.92 & 21.99 & 37.67 & 47.67 & 46.33 & 55.67 & 6.06 & 0.78 & 2.33 & 10.46 & 10.70 & 0.18 & 0.08 & 1.84 \\
\hline 'Calypso’ & 18.13 & 0.00 & 9.10 & 22.94 & 37.33 & 48.00 & 47.00 & 55.67 & 5.60 & 0.82 & 2.74 & 10.75 & 10.75 & 0.17 & 0.06 & 1.76 \\
\hline Mean & 126.44 & 2.08 & 21.85 & 77.02 & 30.50 & 40.46 & 39.10 & 48.56 & 9.32 & 1.89 & 5.51 & 14.34 & 13.91 & 0.77 & 0.12 & 7.75 \\
\hline F-LSD $D_{0.05}$ & 35.72 & 1.39 & 5.39 & 53.98 & 1.55 & 2.08 & 1.90 & 3.41 & 4.43 & 1.50 & 2.33 & 4.95 & 4.35 & 0.72 & 0.07 & 1.83 \\
\hline
\end{tabular}

VL8WAP=Vine length at 8 weeks after planting $(\mathrm{cm})$, LA8WAP=Leaf area at 8 weeks after planting $\left(\mathrm{cm}^{2}\right)$, NoB8WAP=Number of branches at 8 weeks after planting, NoL8WAP=Number of leaves at 8 weeks after planting, DMFI=Days to male flower initiation, DFFI=Days to female flower initiation, $\mathrm{D} 50 \% \mathrm{MF}=\mathrm{Days}$ to $50 \%$ male flowering, $\mathrm{D} 50 \% \mathrm{FF}=$ Days to $50 \%$ female flowering, $\mathrm{NoPFPP}=$ Number of pistillate flower per plant, NoSFPP=Number of staminate flower per plant, $\mathrm{FG}=\mathrm{Fruit}$ Girth (cm), $\mathrm{FL}=\mathrm{Fruit}$ length (cm), FWPP=Fruit weight per plant $(\mathrm{kg}), \mathrm{AFW}=$ Average fruit weight $(\mathrm{kg}), \mathrm{NoFPP}=$ Number of fruit per plant, $\mathrm{TFY} / \mathrm{Ha}=$ Total fruit yield per hectare (tons/ha)

Table 5. Seasonal performance of cucumber genotypes with respect to some vegetative and yield traits evaluated in 2014 planting season

\begin{tabular}{|c|c|c|c|c|c|c|c|c|}
\hline Seasons & VL8WAP & NoB8WAP & NoL8WAP & LA8WAP & DMFI & DFFI & D50\%FM & $\mathrm{D} 50 \% \mathrm{FF}$ \\
\hline Early & 143.67 & 2.16 & 24.81 & 89.66 & 28.52 & 38.33 & 36.96 & 46.75 \\
\hline Late & 126.44 & 2.08 & 21.85 & 77.02 & 30.50 & 40.46 & 39.10 & 48.56 \\
\hline F-LSD 0.05 & 6.22 & ns & 1.05 & 9.41 & 0.38 & 0.52 & 0.44 & 0.66 \\
\hline Seasons & NoSFPP & NoPFPP & NoFPP & FL & FG & FWPP & AFW & TFY/ha \\
\hline Early & 12.50 & 3.56 & 6.40 & 15.58 & 15.03 & 0.96 & 0.18 & 9.61 \\
\hline Late & 9.32 & 1.89 & 5.51 & 14.34 & 13.91 & 0.77 & 0.12 & 7.75 \\
\hline F-LSD 0.05 & 0.79 & 0.29 & 0.44 & 0.88 & 0.77 & 0.13 & 0.03 & 0.02 \\
\hline
\end{tabular}

VL8WAP=Vine length at 8 weeks after planting $(\mathrm{cm}), \mathrm{LA} 8 \mathrm{WAP}=$ Leaf area at 8 weeks after planting $\left(\mathrm{cm}^{2}\right)$, NoB8WAP=Number of branches at 8 weeks after planting, NoL8WAP=Number of leaves at 8 weeks after planting, DMFI=Days to male flower initiation, DFFI=Days to female flower initiation, D50\%MF=Days to $50 \%$ male flowering, D $50 \% \mathrm{FF}=$ Days to $50 \%$ female flowering, NoPFPP=Number of pistillate flower per plant, NoSFPP=Number of staminate flower per plant, FG=Fruit Girth (cm), FL=Fruit length (cm), FWPP=Fruit weight per plant $(\mathrm{kg}), \mathrm{AFW}=$ Average fruit weight $(\mathrm{kg}), \mathrm{NoFPP}=$ Number of fruit per plant, TFY/Ha=Total fruit yield per hectare (tons/ha)

variation among the genotypes for all the traits studied in the early season experiments. In the early season planting, vine length varied significantly from $18.41 \mathrm{~cm}$ for 'Royal F1' to $299.72 \mathrm{~cm}$ for 'Ashely'. The highest number of branches was observed in 'Beit Alpha' which was significantly $(p<0.05)$ higher than the other genotypes except 'Ashely', 'Straight 8', 'Sumter' and 'Centriolo'. 'Sumter' gave the highest number of leaves, followed by 'Ashely' and 'Centriolo' while the least number of leaves occurred in 'MarketMore 76'. 'Sumter' gave the highest leaf area that was significantly $(\mathrm{p}<0.05)$ higher than the other genotypes with the exception of 'Regal' and 'Ashely'. 
Table 6. Correlation coefficient for 15 traits of cucumber genotypes evaluated in 2014 early planting season

\begin{tabular}{|c|c|c|c|c|c|c|c|c|c|c|c|c|c|c|c|}
\hline ATTRIBUTES & $\begin{array}{c}\text { VL8 } \\
\text { WAP }\end{array}$ & $\begin{array}{l}\text { NoB8 } \\
\text { WAP }\end{array}$ & $\begin{array}{l}\text { NoL8 } \\
\text { WAP }\end{array}$ & $\begin{array}{c}\text { LA8 } \\
\text { WAP }\end{array}$ & DMFI & DFFI & $\begin{array}{c}\text { D50\% } \\
\text { MF }\end{array}$ & $\begin{array}{c}550 \% \\
\text { FF }\end{array}$ & $\begin{array}{c}\text { NoSF } \\
\text { PP }\end{array}$ & $\begin{array}{c}\text { NoPF } \\
\text { PP }\end{array}$ & $\begin{array}{l}\text { NoF } \\
\text { PP }\end{array}$ & $\mathrm{FL}$ & FG & $\begin{array}{l}\mathrm{FW} \\
\text { PP }\end{array}$ & TFY/ha \\
\hline \multicolumn{16}{|c|}{ Early Season Planting } \\
\hline VLSWAP & 1 & $.888^{* *}$ & $.928^{* *}$ & $.804^{* *}$ & $-.623^{* *}$ & $-.634^{* *}$ & $-.617^{* *}$ & $-597^{* *}$ & $.774^{* *}$ & $.753^{* *}$ & $.846^{* *}$ & $.677^{* *}$ & $.652^{* *}$ & $.763^{* *}$ & $.742^{* *}$ \\
\hline NoB8WAP & & 1 & $.882^{* *}$ & $.845^{* *}$ & $-597^{* *}$ & $-613^{* *}$ & $-590^{* *}$ & $-.579 *$ & $.798^{* *}$ & $.791^{* *}$ & $.878^{* *}$ & $.690^{* *}$ & $.650^{* *}$ & $.795^{* *}$ & $.756^{* *}$ \\
\hline NoL8WAP & & & 1 & $.870^{* *}$ & $-.627^{* *}$ & $-648^{* *}$ & $-.623^{* *}$ & $-585^{* *}$ & $.841^{* *}$ & $.830^{* *}$ & $.908^{* *}$ & $.752^{* *}$ & $.718^{* *}$ & $.827^{* *}$ & $.776^{* *}$ \\
\hline LA8WAP & & & & 1 & $-.726^{* *}$ & $-.756^{* *}$ & $-.725^{* *}$ & $-.677^{* *}$ & $.984^{* *}$ & $.974^{* *}$ & $.966^{* *}$ & $.937^{* *}$ & $.904^{* *}$ & $.983^{* *}$ & $.953^{* *}$ \\
\hline DMFI & & & & & 1 & $.984^{* *}$ & $.990^{* *}$ & $.928^{* *}$ & $-700^{* *}$ & $-.658^{* *}$ & $-.752^{* *}$ & $-722^{* *}$ & $-749^{* *}$ & $-.669^{* *}$ & $-.622^{* *}$ \\
\hline DFFI & & & & & & 1 & $.980^{* *}$ & $.927^{* *}$ & $-.734^{* *}$ & $-703^{* *}$ & $-.761^{* *}$ & $-.734^{* *}$ & $-.752^{* *}$ & $-.708^{* *}$ & $-.661^{* *}$ \\
\hline $\mathrm{D} 50 \% \mathrm{MF}$ & & & & & & & 1 & $.930^{* *}$ & $-.697^{* *}$ & $-.657^{* *}$ & $-.743^{* *}$ & $-.721^{* *}$ & $-.749^{* *}$ & $-.669^{* *}$ & $-.624^{* *}$ \\
\hline $\mathrm{D} 50 \% \mathrm{FF}$ & & & & & & & & 1 & $-.646^{* *}$ & $-.611^{* *}$ & $-.705^{* *}$ & $-.668^{* *}$ & $-708^{* *}$ & $-.625^{* *}$ & $-.574^{* *}$ \\
\hline NoSFPP & & & & & & & & & 1 & $.987^{* *}$ & $.940^{* *}$ & $.940^{* *}$ & $.913^{* *}$ & $.978^{* *}$ & $.949^{* *}$ \\
\hline NoPFPP & & & & & & & & & & 1 & $.923^{* *}$ & $.926^{* *}$ & $.894^{* *}$ & $.980^{* *}$ & $.949^{* *}$ \\
\hline NoFPP & & & & & & & & & & & 1 & $.906^{* *}$ & $.880^{* *}$ & $.923^{* *}$ & $.886^{* *}$ \\
\hline FL & & & & & & & & & & & & 1 & $.979^{* *}$ & $.938^{* *}$ & $.911^{* *}$ \\
\hline FG & & & & & & & & & & & & & 1 & $.899^{* *}$ & $.883^{* *}$ \\
\hline FWPP & & & & & & & & & & & & & & 1 & $.978^{* *}$ \\
\hline TFY/ha & & & & & & & & & & & & & & & 1 \\
\hline \multicolumn{15}{|c|}{${ }^{*}$ Correlation is significant at the 0.01 level } & \\
\hline
\end{tabular}

VL8WAP=Vine length at 8 weeks after planting (cm), LA8WAP=Leaf area at 8 weeks after planting (cm2), NoB8WAP=Number of branches at 8 weeks after planting, NoL8WAP=Number of leaves at 8 weeks after planting, DMFI=Days to male flower initiation, DFFI=Days to female flower initiation, D50\%MF=Days to $50 \%$ male flowering, $\mathrm{D} 50 \% \mathrm{FF}=$ Days to $50 \%$ female flowering, NoPFPP=Number of pistillate flower per plant, NoSFPP=Number of staminate flower per plant, FG=Fruit Girth (cm), FL=Fruit length $(\mathrm{cm}), \mathrm{FWPP}=$ Fruit weight per plant $(\mathrm{kg}), \mathrm{AFW}=$ Average fruit weight $(\mathrm{kg}), \mathrm{NoFPP}=$ Number of fruit per plant, $\mathrm{TFY} / \mathrm{Ha}=\mathrm{Total}$ fruit yield per hectare (tons/ha)

Table 7. Correlation coefficient for 15 traits of cucumber genotypes evaluated in 2014 late planting season

\begin{tabular}{|c|c|c|c|c|c|c|c|c|c|c|c|c|c|c|c|}
\hline ATTRIBUTES & $\begin{array}{l}\text { VL8 } \\
\text { WAP }\end{array}$ & $\begin{array}{l}\text { NoB8 } \\
\text { WAP }\end{array}$ & $\begin{array}{l}\text { NoL8 } \\
\text { WAP }\end{array}$ & $\begin{array}{c}\text { LA8 } \\
\text { WAP }\end{array}$ & DMFI & DFFI & $\begin{array}{l}\mathrm{D} 50 \% \\
\mathrm{MF}\end{array}$ & $\begin{array}{c}\text { D50\% } \\
\text { FF }\end{array}$ & $\begin{array}{l}\text { NoSF } \\
\text { PP }\end{array}$ & $\begin{array}{c}\text { NoPF } \\
\text { PP }\end{array}$ & $\begin{array}{l}\mathrm{NoF} \\
\mathrm{PP}\end{array}$ & FL & FG & $\begin{array}{l}\mathrm{FW} \\
\mathrm{PP}\end{array}$ & TFY/ha \\
\hline \multicolumn{16}{|c|}{ Late Season Planting } \\
\hline VL8WAP & 1 & $.908^{* *}$ & $.930^{* *}$ & $.810^{* *}$ & $-.643^{* *}$ & $-.651^{* *}$ & $-.641^{* *}$ & $-.548^{* *}$ & $.797^{* *}$ & $.805^{* *}$ & $.824^{* *}$ & $.919^{* *}$ & $.897^{* *}$ & $.903^{* *}$ & $.862^{* *}$ \\
\hline NoB8WAP & & 1 & $.867^{* *}$ & $.748^{* *}$ & $-.608^{* *}$ & $-.618^{* *}$ & $-.614^{* *}$ & $-.519^{* *}$ & $.732^{* *}$ & $.716^{* *}$ & $.769^{* *}$ & $.795^{* *}$ & $.775^{* *}$ & $.815^{* *}$ & $.764^{* *}$ \\
\hline NoL8WAP & & & 1 & $.842^{* *}$ & $-.627^{* *}$ & $-.636^{* *}$ & $-.619^{* *}$ & $-.453^{* *}$ & $.768^{* *}$ & $.749^{* *}$ & $.778^{* *}$ & $.872^{* *}$ & $.856^{* *}$ & $.783^{* *}$ & $.726^{* *}$ \\
\hline LA8WAP & & & & 1 & $-.776^{* *}$ & $-.759^{* *}$ & $-.774^{* *}$ & $-.640^{* *}$ & $.911^{* *}$ & $.871^{* *}$ & $.891^{* *}$ & $.812^{* *}$ & $.818^{* *}$ & $.713^{* *}$ & $.710^{* *}$ \\
\hline DMFI & & & & & 1 & $.980^{* *}$ & $.984^{* *}$ & $.885^{* *}$ & $-.718^{* *}$ & $-.726^{* *}$ & $-.825^{* *}$ & $-.741^{* *}$ & $-.790^{* *}$ & $-.721^{* *}$ & $-.742^{* *}$ \\
\hline DFFI & & & & & & 1 & $.974^{* *}$ & $.887^{* *}$ & $-.704^{* *}$ & $-.723^{* *}$ & $-.822^{* *}$ & $-.735^{* *}$ & $-.785^{* *}$ & $-.730^{* *}$ & $-.741^{* *}$ \\
\hline D $50 \% \mathrm{MF}$ & & & & & & & 1 & $.900^{* *}$ & $-.705^{* *}$ & $-.712^{* *}$ & $-.815^{* *}$ & $-.746^{* *}$ & $-.796^{* *}$ & $-.705^{* *}$ & $-.725^{* *}$ \\
\hline D50\%FF & & & & & & & & 1 & $-.585^{* *}$ & $-.584^{* *}$ & $-.716^{* *}$ & $-.630^{* *}$ & $-.682^{* *}$ & $-.602^{* *}$ & $-.607^{* *}$ \\
\hline NoSFPP & & & & & & & & & 1 & $.939^{* *}$ & $.950^{* *}$ & $.816^{* *}$ & $.795^{* *}$ & $.768^{* *}$ & $.787^{* *}$ \\
\hline NoPFPP & & & & & & & & & & 1 & $.944^{* *}$ & $.834^{* *}$ & $.819^{* *}$ & $.807^{* *}$ & $.820^{* *}$ \\
\hline NoFPP & & & & & & & & & & & 1 & $.871^{* *}$ & $.857^{* *}$ & $.823^{* *}$ & $.841^{* *}$ \\
\hline FL & & & & & & & & & & & & 1 & $.977^{* *}$ & $.891^{* *}$ & $.875^{* *}$ \\
\hline FG & & & & & & & & & & & & & 1 & $.883^{* *}$ & $.866^{* *}$ \\
\hline FWPP & & & & & & & & & & & & & & 1 & $.964^{* *}$ \\
\hline TFY/ha & & & & & & & & & & & & & & & 1 \\
\hline $\begin{array}{l}{ }^{* *} \text { Correlation is sig } \\
{ }^{*} \text { Correlation is sign }\end{array}$ & $\begin{array}{l}\text { ficant at th } \\
\text { icant at the }\end{array}$ & $\begin{array}{l}0.01 \text { level } \\
.05 \text { level }\end{array}$ & & & & & & & & & & & & & \\
\hline
\end{tabular}

VL8WAP=Vine length at 8 weeks after planting $(\mathrm{cm})$, LA8WAP=Leaf area at 8 weeks after planting $\left(\mathrm{cm}^{2}\right)$, NoB8WAP=Number of branches at 8 weeks after planting, NoL8WAP=Number of leaves at 8 weeks after planting, DMFI=Days to male flower initiation, DFFI=Days to female flower initiation, D50\%MF=Days to $50 \%$ male flowering, $\mathrm{D} 50 \% \mathrm{FF}=$ Days to $50 \%$ female flowering, NoPFPP=Number of pistillate flower per plant, NoSFPP=Number of staminate flower per plant, FG=Fruit Girth (cm), FL=Fruit length (cm), FWPP=Fruit weight per plant (kg), AFW=Average fruit weight $(\mathrm{kg}), \mathrm{NoFPP}=\mathrm{Number}$ of fruit per plant, TFY/Ha=Total fruit yield per hectare $($ tons $/ \mathrm{ha})$

'Beit Alpha', 'Ashely' and 'Regal' each recorded the lowest number of days to male flower initiation. Also, 'Beit Alpha', 'Ashely', 'Regal' and 'Sumter' gave the lowest number of days to female flower initiation. The least days to $50 \%$ male flowering was observed in 'Regal' which was statistically the same with 'Ashely', 'Beit Alpha', 'Straight 8' and 'Sumter' but significantly $(\mathrm{p}<0.05)$ different from the rest. The lowest number of days to $50 \%$ female flowering was recorded in 'Regal' followed by 'Ashely', 'Beit Alpha' and 'Straight 8' which were statistically the same. The highest number of staminate flower per plant was obtained from 'Sumter' which was the same statistically with 'Straight 8 ' but significantly $(\mathrm{p}<0.05)$ higher when compared to other genotypes (Table 3). Number of pistillate flower per plant varied significantly $(\mathrm{p}<0.05)$ from 1.91 for 'Marketer' to 6.87 for 'Straight 8 ' which was statistically the same with 'Sumter'. The highest number of fruits per plant was recorded in 'Straight 8 ' which was statistically the same when compared to 'Sumter' and 'Ashely'. Fruit length of the genotypes showed significant $(p<0.05)$ differences. 'Straight 8 ' had the highest fruit length, followed by 'Beit Alpha' and 'Ashely' which were statistically the same. The least fruit length was observed in 'MarketMore 76 . Significantly $(p<0.05)$, highest fruit girth and fruit weight per plant were observed in the 'Straight 8'. However, the least fruit girth and fruit weight per plant were obtained in 'Delilha' and 'Royal F1' respectively. Average fruit weight was significantly $(\mathrm{p}<0.05)$ higher in 'Poinsett' than the rest genotypes. 'Regal' had the least average fruit weight. 'Beit Alpha' showed significantly $(\mathrm{p}<0.05)$ the highest total fruit yield per hectare (Table 3).

The performance of cucumber genotypes with respect to some agronomic traits evaluated in 2014 late season planting is presented in Table 4. The result showed a significant $(p<0.05)$ variation among the genotypes for all the traits 
Table 8. Direct and indirect effects of traits on yield in cucumber genotypes evaluated in 2014 both early and late planting season

\begin{tabular}{|c|c|c|c|c|c|c|c|c|c|c|c|c|c|}
\hline \multicolumn{14}{|c|}{ Early Season Planting } \\
\hline & VL8WAP & NoB8WAP & NoL8WAP & DMFI & DFFI & NoSF & NoPF & $\mathrm{NoF}$ & FL & FG & $\mathrm{FW}$ & TFY & TIE \\
\hline VL8WAP & 0.244 & -0.064 & -0.271 & -0.166 & 0.102 & 0.054 & -0.174 & 0.103 & -0.039 & 0.080 & 0.874 & $0.742^{* *}$ & 0.499 \\
\hline NoB8WAP & 0.217 & -0.072 & -0.258 & -0.159 & 0.098 & 0.055 & -0.183 & 0.107 & -0.039 & 0.079 & 0.911 & $0.756^{* *}$ & 0.828 \\
\hline NoL8WAP & 0.226 & -0.064 & -0.292 & -0.167 & 0.104 & 0.059 & -0.192 & 0.111 & -0.043 & 0.088 & 0.947 & $0.776^{* *}$ & 1.069 \\
\hline DMFI & -0.152 & 0.043 & 0.183 & 0.267 & -0.158 & -0.049 & 0.152 & -0.092 & 0.041 & -0.092 & -0.767 & $-0.622^{* *}$ & -0.889 \\
\hline DFFI & -0.155 & 0.044 & 0.189 & 0.263 & -0.160 & -0.051 & 0.162 & -0.093 & 0.042 & -0.092 & -0.811 & $-0.661^{* *}$ & -0.821 \\
\hline NoSF & 0.189 & -0.058 & -0.246 & -0.187 & 0.118 & 0.069 & -0.228 & 0.114 & -0.054 & 0.112 & 1.120 & $0.949^{* *}$ & 0.880 \\
\hline NoPF & 0.184 & -0.057 & -0.242 & -0.176 & 0.113 & 0.068 & -0.231 & 0.112 & -0.053 & 0.109 & 1.122 & $0.949^{* *}$ & 0.180 \\
\hline $\mathrm{NoF}$ & 0.206 & -0.063 & -0.265 & -0.201 & 0.122 & 0.065 & -0.213 & 0.122 & -0.052 & 0.108 & 1.057 & $0.886^{* *}$ & 0.764 \\
\hline FL & 0.165 & -0.050 & -0.220 & -0.193 & 0.118 & 0.065 & -0.214 & 0.110 & -0.057 & 0.120 & 1.074 & $0.919^{* *}$ & 0.976 \\
\hline FG & 0.159 & -0.047 & -0.210 & -0.200 & 0.121 & 0.063 & -0.207 & 0.107 & -0.056 & 0.122 & 1.029 & $0.883^{* *}$ & 0.761 \\
\hline FW & 0.186 & -0.057 & -0.241 & -0.179 & 0.114 & 0.068 & -0.226 & 0.112 & -0.054 & 0.110 & 1.145 & $0.978^{* *}$ & -0.167 \\
\hline \multicolumn{14}{|c|}{ Residual $=0.030$} \\
\hline VL8WAP & 0.190 & -0.049 & -0.233 & 0.099 & -0.060 & 0.068 & -0.012 & 0.059 & 0.099 & -0.035 & 0.736 & $0.862^{* *}$ & 0.672 \\
\hline NoB8WAP & 0.173 & -0.054 & -0.218 & 0.094 & -0.057 & 0.063 & -0.010 & 0.055 & 0.086 & -0.031 & 0.664 & $0.764^{* *}$ & 0.818 \\
\hline NoL8WAP & 0.177 & -0.047 & -0.251 & 0.097 & -0.059 & 0.066 & -0.011 & 0.056 & 0.094 & -0.034 & 0.638 & $0.726^{* *}$ & 0.977 \\
\hline DMFI & -0.122 & 0.033 & 0.157 & -0.154 & 0.090 & -0.061 & 0.011 & -0.059 & -0.080 & 0.031 & -0.588 & $-0.742^{* *}$ & -0.588 \\
\hline DFFI & -0.124 & 0.034 & 0.160 & -0.151 & 0.092 & -0.060 & 0.011 & -0.059 & -0.079 & 0.031 & -0.595 & $-0.741^{* *}$ & -0.833 \\
\hline NoSF & 0.152 & -0.040 & -0.193 & 0.111 & -0.065 & 0.085 & -0.014 & 0.068 & 0.088 & -0.031 & 0.625 & $0.787^{* *}$ & 0.702 \\
\hline NoPF & 0.153 & -0.039 & -0.188 & 0.112 & -0.067 & 0.080 & -0.015 & 0.067 & 0.090 & -0.032 & 0.657 & $0.820^{* *}$ & 0.835 \\
\hline $\mathrm{NoF}$ & 0.157 & -0.042 & -0.195 & 0.127 & -0.076 & 0.081 & -0.014 & 0.071 & 0.094 & -0.034 & 0.671 & $0.841^{* *}$ & 0.770 \\
\hline FL & 0.175 & -0.043 & -0.219 & 0.114 & -0.068 & 0.070 & -0.012 & 0.062 & 0.108 & -0.039 & 0.726 & $0.875^{* *}$ & 0.767 \\
\hline FG & 0.171 & -0.042 & -0.215 & 0.122 & -0.072 & 0.068 & -0.012 & 0.061 & 0.105 & -0.039 & 0.719 & $0.866^{* *}$ & 0.905 \\
\hline FW & 0.172 & -0.044 & -0.197 & 0.111 & -0.067 & 0.066 & -0.012 & 0.059 & 0.096 & -0.035 & 0.815 & $0.964^{* *}$ & 0.149 \\
\hline
\end{tabular}

VL8WAP=Vine length at 8 weeks after planting $(\mathrm{cm})$, LA8WAP=Leaf area at 8 weeks after planting $\left(\mathrm{cm}^{2}\right)$, NoB8WAP=Number of branches at 8 weeks after planting, NoL8WAP=Number of leaves at 8 weeks after planting, DMFI=Days to male flower initiation, DFFI=Days to female flower initiation, D50\%MF=Days to $50 \%$ male flowering, $\mathrm{D} 50 \% \mathrm{FF}=$ Days to $50 \%$ female flowering, NoPFPP=Number of pistillate flower per plant, NoSFPP=Number of staminate flower per plant, FG=Fruit Girth (cm), FL=Fruit length (cm), FWPP=Fruit weight per plant (kg), AFW=Average fruit weight $(\mathrm{kg}), \mathrm{NoFPP}=$ Number of fruit per plant, TFY/Ha=Total fruit yield per hectare (tons/ha)

studied. 'Ashely' had significantly $(\mathrm{p}<0.05)$ the highest values in all the traits studied in late season planting except in number of leaves where 'Sumter' took the lead though statistically similar to 'Ashely' (Table 4). However, the least days to male and female flower initiation and days to $50 \%$ male flowering were shared between 'Ashely' and 'Beit Alpha' except in days to $50 \%$ female flowering where 'Beit Alpha' recorded the least value though statistically the same.

The combined analysis of variance of the two seasons planting showed that all the genotypes performed significantly $(p<0.05)$ higher during the early rains in all the traits studied than the late except number of branches though higher in early season planting (Table 5).

The result of the correlation coefficient among some agronomic traits of cucumber genotypes evaluated in early season planting is presented in Table 6. From the correlation matrix, all the traits showed high significant $(\mathrm{p}<0.01)$ correlations with total fruit yield/ha. Vine length, number of branches, number of leaves, leaf area, number of staminate flower per plant, number of pistillate flower per plant, number of fruits per plant, fruit length, fruit girth, and fruit weight per plant gave positive correlation with total fruit yield while days to male and female flower initiation, days to $50 \%$ male and female flowering were negatively correlated with total fruit yield and the rest traits. Similar result was obtained in the late rainy season planting (Table 7).

The results of the path coefficient analysis of some agronomic traits in cucumber genotypes evaluated in 2014 early and late rainy season planting are presented in Table 8.

In both planting seasons; vine length, number of staminate flower per plant, number of fruit per plant and fruit weight per plant had positive direct effects on the total fruit yield/ha. Also, number of branches, number of leaves and number of pistillate flower per plant consistently gave negative direct effects on the total fruit yield/ha. Fruit weight per plant constantly gave the highest positive direct effect on total fruit yield/ha in both seasons while the highest negative direct effect was obtained from number of leaves in both seasons. In early season planting, the indirect effects of fruit weight per plant through vine length, days to male flower initiation, number of staminate flower per plant, number of fruit per plant and fruit girth were positive while its pathway through number of branches, number of leaves, days to female flower initiation, number of pistillate flower per plant and fruit length were negative. Similar trend was observed in the late season planting with the exception of days to female flower initiation and fruit length that gave positive indirect effects and days to male flower initiation and fruit girth that gave negative indirect effects. The residual factor obtained in both early and late seasons planting were 0.03 and 0.05 respectively (Table 8 ).

\section{Discussion}

In the characterization study, the significant differences observed among the cucumber genotypes in all the attributes studied in both early and late rainy seasons planting showed that considerable variation existed in the genotypes. In early season planting, the superior performance of 'Beit Alpha', 'Straight 8, 'Ashely' and 'Sumter' in total fruit yield/ha can be attributed to their longer vines, higher numbers of branches and leaves per plant. The early activation of the reproductive phase was pivotal in translocation of the photosynthates to the sink (fruit) thereby increasing the fruit yield (Sonia et al., 2014). The absence of high number of fruits by 'Poinsett' in 
the early season planting may be responsible for high average fruit weight obtained as most assimilates were deposited into fewer sinks. In the late season planting, 'Ashely', 'Sumter' and 'Beit Alpha' maintained their superior performance in most of the traits studied including total fruit yield/ha. This findings aligned with Gichimu et al. (2008) where high vine length, number of branches and leaves have been shown to produce higher yields than those with fewer branches in watermelon.

The consistent superior performance of these genotypes across environments is an indication to their genetic makeup. Their ability to perform well in a new environment showed little environmental influence. Yield is a complex quantitative trait, considerably affected by environment; therefore, selection of genotypes based on yield per se is not effective (Cetin et al., 2009). Hence, selecting for yield components would reduce the environmental influence on the yield (Uguru, 1995).

Correlation of particular traits with other traits and with yield is important in indirect selection of genotypes for yield improvement (Machikowa and Laosuwan, 2011). Significant and positive correlation between two characters suggests that these characters can be improved simultaneously in a selection programme (Hayes et al., 1955; Fayeun et al., 2012). This is because it shows mutual relationship among characters and selection for one will translate to selection and improvement of the other (Fayeun et al., 2012). The result in both seasons indicated that all the traits except days to flower appearances correlated positively with total fruit yield/ha. This implies that selection for such traits would result to higher total fruit yield per hectare. This is in agreement with those of Islam et al. (1993) and Cramer and Wehner (2000) who reported significant positive correlation between these traits and yield in cucumber. Negative correlations between all the days to flower appearances and fruit yield suggest earliness or lateness to flower initiation and fruit maturity which would have an inverse effect on yield (Cramer and Wehner, 2000). Ramirez et al. (1988) and Afangideh et al. (2005) also observed significant positive correlations between number of fruits/plant, stem length and total fruit yield/ha in cucumber. A strong positive and significant relationship between number of fruit/plant, fruit diameter and flesh thickness and total fruit yield/ha have also been reported in cucumber (Ullah et al., 2012). Negative correlation between days to flower appearances and total fruit yield per hectare in both seasons showed that high fruit yield was a function of least number of days to flower appearance which resulted to earliness in fruit maturity. The finding is in line with those of Afangideh and Uyoh (2007) and Ogbodo et al. (2010) on cucumber. Number of fruits per plant and fruit weight showed significant and positive correlation with total fruit yield. The finding is in consonance with results of Golabadi et al. (2013) in their studies on determining relationships between different horticultural traits in Cucumis sativus L. genotypes.

Basing selection decision on correlation coefficient alone may give a misleading impression as it only measures the degree of mutual association between two variables without regard to causation. This is because there is the risk of omitting some useful traits whose contributions through other traits might not be easily appreciated. It is important to measure the mutual relationship between various plant attributes and determine the component characters, on which selection procedure can be based for direct and indirect genetic improvement of crop yield (Hassan et al., 2013). In the present study, in both early and late rainy seasons planting, fruit weight/plant exerted the highest direct effect on total fruit yield/ha. It suggests therefore, that, this trait (fruit weight/plant) is the most important trait contributing to variability in yield improvement. Similar result had been reported by Nwofia et al. (2015) in cucumber. However, in both seasons planting; vine length, number of staminate flower per plant, number of fruit per plant and fruit weight per plant had positive direct effects on the total fruit yield/ha. It shows that increase in yield may depend solely on direct selection of these traits. Also, number of branches, number of leaves and number of pistillate flower per plant consistently gave negative direct effects on the total fruit yield/ha. It gives an indication that direct selection of these traits may not increase yield. Hence, selection for these traits must be done indirectly via fruit weight/plant (Ndukauba et al., 2015). Uguru (1996) and Nwofia et al. (2015) opined that it is an indication that the traits have appreciable value despite the negative direct effects recorded. The residual factors which determine the extent to which the casual factors have explained the variability in fruit yield were low and observed to be 0.030 and 0.052 in early and late season plantings respectively. This is an indication that $97 \%$ and $95 \%$ of the total variability in total fruit yield/ha had been sufficiently accounted for by the traits that were used in the path analysis in both season plantings. Comparable residual factors had been reported in cucumber (Nwofia et al., 2015).

\section{Conclusions}

Significantly, the highest total fruit yield/ha was recorded in the early rainy season planting. 'Beit Alpha' genotype gave the highest fruit yield in early season planting while in late season, Ashley took the lead. These genotypes are recommended for cultivation in the Derived Savannah, Southeast Nigeria agro ecological zone. Path coefficients analysis revealed that fruit weight per plant having the highest positive direct effect on yield is of utmost importance in contributing to yield improvement in both seasons and hence, requires major concern.

\section{References}

Afangideh U, Uyoh EA (2007). Genetic variability and correlation studies in some varieties of cucumber (Cucumis sativus $\mathrm{L}$.). Jordan Journal of Agricultural Science 3(4):376-384.

Afangideh, U, Uyoh EA, Ittah M, Uko AE (2005). Morphological characterization of some cultivars of cucumber (Cucumis sativus L.). Journal of Sustainable Tropical Agricultural Research 14:1318.

Ajisefinanni A (2004). Performance of two cucumber varieties in response to manure rates and types at Samaru. Undergraduate project Agronomy Dept ABU Zaria, Nigeria.

Anonymous (2012). Nutritional recommendations for cucumber (Cucumis sativus L.) in the open fields, tunnel and greenhouse. Haifa, Pioneering the Future pp 4-5. http://www.haifagroup.com/files/Guides/Cucumber.pdf. 
Cetin K, Emine K, Remzi E, Oktay G (2009). Correlations and path coefficient analysis between leaf chlorophyll content, yield and yield components in cotton (Gossypium hirsutum L.) under drought stress conditions. Notulae Botanicae Horti Agrobotanici Cluj-Napoca 37(2):241-244.

Cramer CS, Wehner TC (2000). Fruit yield and yield component correlations of four pickling cucumber populations. Cucurbit Genetics Cooperative Report 23:12-15.

Dewey DR, Lu KH (1959). A correlation and path coefficient analysis of components of crested wheat grass seed production. Agronomy Journal (75):153-155.

Eifediyi EK, Remison SU (2009). Effect of time of planting on the growth and yield of five varieties of cucumber. Report and Opinion 1(5):81-90.

Eifediyi EK, Remison SU (2010). Growth and yield of cucumber (Cucumis sativus L.) as influenced by farmyard manure and inorganic fertilizer. Journal of Plant Breeding and Crop Science 2(7):216-220.

Eneobong HN (2001). Eating Right. A nutrition guide. University of Calabar press, Calabar.

ErnestinaP(2001). Cuaumber.http.www.earthnotestripod.com/auaumberhtml.

Fayeun LS, Odiyi AC, Makinde SCO, Aiyelari OP (2012). Genetic variability and correlation studies in the fluted pumpkin (Telfairia occidentalis Hook F.). Journal of Plant Breeding and Crop Science 4(10):156-160.

Gichimu BM, Owuor BO, Dida MM (2008). Agronomic performance of three most popular commercial watermelon cultivars in Kenya as compared to one newly introduced cultivar and one local landrace grown on dystricnitisols under sub-humid tropical conditions. ARPN Journal of Agricultural and Biological Science (3-5-6):65-71.

Golabadi M, Eghtedary AR, Golkar PP (2013). Determining relationships between different horticultural traits in (Cucumis sativus L.) Genotypes with multivariate analysis. Sabrao Journal of Breeding and Genetics (45)3:447-457.

Griffing B (1956). Concept of general and specific combining ability in relation to diallel crossing system. Australian Journal of Biological Sciences 9:463-493.

Hassan SMF, Muhammad SI, Ghulam R, Naeem-ud-Din, Ghulam S, Muhammad R, Ijaz RN (2013). Correlation and path analysis for yield and yield components in sunflower (Helianthus annus L). African Journal of Biotechnology 12(16):1968-1971.

Hayes HK, Forrest RI, Smith DC (1955). Methods of plant breeding, correlation and regression in relation to plant breeding. McGraw Hill Company Inc. $2^{\text {nd }}$ Edition pp 439-451.

Islam MS, Khan S, Khanem D, Malex A, Hogue AMM (1993).Genetic variability and path analysis in cucumber (Cucumis sativus L.). Bangladesh Journal of Plant Breeding and Genetics (6):45-51.

Jizhe C (1994). Cucumber evaluation trial. ARC/AVRDC Training Thailand.

Lawrence WJC (1968). Plant breeding. The National Academic Press London.

Lower RL, Edwards MD (1986). Cucumber breeding. In: Basset MJ
(Ed). Breeding vegetables crops. Westport, Connecticut USA: AVI Publishing Co pp 173-203.

Machikowa T, Laosuwan P (2011). Path coefficient analysis for yield of early maturing soybean. Songklanakarin Journal of Science and Technology (33)4:365-368.

Manyvong V (1997). Cucumber varietal trial ARC/AVRDC Training workshop, Thailand.

Mingbao L (1991). Cucumber varietal trial.ARC/AVRDC Training Bangkok Thailand.

Ndukauba J, Nwofia GE, Okocha PI, Ene-Obong EE (2015). Variability in Egusi-Melon genotypes (Citrullus lanatus (Thumb) Matsum and Nakai) in Derived Savannah environment in SouthEastern Nigeria. Vegetos-International Journal of Plant Research (5) 1:19-26.

Nwofia GE, Amajuoyi AN, Mbah EU (2015). Response of three cucumber varieties (Cucumis sativus L.) to planting season and NPK fertilizer rates in lowland humid tropics: sex expression, yield and inter-relationships between yield and associated traits. International Journal of Agriculture and Forestry (5)1:30-37.

Obi IU (2002). Statistical methods of detecting difference between treatment means and research methodology issues in laboratory and field experiments, Second edition. Dept. of Crop Sci, University of Nigeria, Nsukka.

Ogbodo EN, Okorie PO, Utobo EB (2010). Introducing cucumber for cultivation at new different zone in Ebonyi State, southeastern, Nigeria. Libyan Agriculture Research Center Journal International (16)1:336-343.

Phu NT (1998). Nitrogen and Potassium effect on cucumber yield. ARC/AVRDC. Training Bangkok Thailand.

Ramirez DR, Wehner TC, Miller CH (1988). Growth analysis and correlation studies in three cucumber lines differing in plant habit. HortScience (23)1:145-148.

Renner SS, Schaefer H, Kocyan A (2007). Phylogenetics of Cucumis (Cucurbitaceae): cucumber (C. sativus) belongs in an Asian/Australian clade far from melon (C. melo). BMC Evolutionary Biology 7:58.

Singh P, Bhatta RS, Singh IP (1977). Genetic variability and path analysis for yield attributes in lentil. Indian Journal of Agricultural Research 11:25-28.

Sonia O, Yong-Ling R, Alisdair RF (2014). An update on source-tosink carbon partitioning in tomato. Frontier in Plant Science 5:516.

Subramanian A, Subbaraman N (2010). Hierarchical cluster analysis of genetic diversity in maize germplasm. European Journal of Pharmaceutics and Biopharmaceutics (1)4:431-436.

Swanbeck G (1968). A new treatment of ichthyosis and other hyperkeratotic condition. Acta Dermato-Venereologica 48:123-7.

Tatlioglu T (1993). Cucumber (Cucumis sativus L.). In: Kallo G, Bergh BO (eds). Genetic improvement of vegetable crops. Pergamon press Ltd.; Tarrytown New York.

Thoa DK (1998). Cucumber seed multiplication and characterisation. AVRDC/ARC Training Thailand.

Uguru MI (1995). Heritability relationships and variability of yield and yield components in vegetable cowpea. African Crop Science Journal 3(1):23-28. 
92

Uguru MI (1996). Estimate of variability and genetic gain in vegetable cowpea (Vigna unguiculata (L.) Walp). Ghana Journal of agricultural Science 29:47-51.

Uguru MI, Baiyeri KP, Abba SC (2011). Indicators of climate change in the derived savannah niche on Nsukka, South-Eastern Nigeria. Journal of Tropical Agriculture, Food, Environment and Extension (10)1:1-10.

Ullah MZ, Hassan MJ, Chowdhury AZMKA, Saki AI, Rahman AHMA (2012). Genetic variability and correlation in exotic cucumber (Cucumis sativus L.) varieties energy pac Agro Ltd. Monipur, Hotapara, Gazipur. Bangladesh Journal of Plant Breeding and Genetics (25)1:17-23.
Uzodike EB, Onuoha IN (2009). The effect of cucumber (Cucumis sativus L.) extract on acid induced corneal burn in Guinea Pigs. African Journal Online 15:3-7.

Wehner TC (2007). Cucumbers, watermelon, squash and other cucurbits. In: Encyclopedia of Food and Culture pp 474-479.

Wehner TC, Guner N (2004). Growth stage, flowering pattern, yield and harvest date prediction of four types of cucumber tested at 10 planting dates. Acta Horticulturae 637:223-230.

Whitaker TW, Davis GN (1996). Cucurbits-botany, cultivation, utilization. Interscience Publ, New York. 